\title{
My patient wants to record our appointment, what should I do?
}

\author{
A patient's request to record a consultation might leave you feeling uneasy but a recording can be \\ a useful tool to empower patients, Abi Rimmer finds
}

Abi Rimmer

The BMJ

\section{Recordings can aid understanding}

Kiersty Griffiths, standards policy manager for the General Medical Council, says, "The idea may be daunting at first but recordings can be a great tool for doctors and patients looking to overcome barriers to communication and understanding.

"For instance, a patient's concentration may be hampered by unspoken anxieties or pain, making it difficult to recall detail. Some patients need extra support because of unfamiliarity with the language or concepts discussed.

"Recordings give patients more time to process the sometimes complex information provided during consultations. They can also help patients to involve their family or carers in decisions about their treatment.

"The information in the consultation is the patient's and-providing no other patient is involved-it's perfectly legal for them to record it for their own use.

"We understand that, if announced in a challenging way, you may feel uncomfortable with a patient's decision to make a recording.

"Where this is the case, you should explore this with the patient in a polite, considerate, and fair way. You shouldn't feel pressured to treat or prescribe any differently than you would usually. It's important that patients understand the risks and benefits of their treatment so they can make informed decisions about their care, so you should continue to give them all the information they might need or want.

"Doctors who follow our core guidance, and act in professional way, should feel assured that this shouldn't raise any concerns."

\section{"It's not a sign of mistrust"}

Roopinder Brar, GP and medical adviser for the Medical and Dental Defence Union of Scotland, says, "It is not uncommon for patients to ask to record consultations and they don't need a doctor's consent to do so. Under data protection regulations the information in the recording belongs to them and they have the right to use it as they choose.

"While this may be unsettling for some doctors, it's important to avoid defensive reactions and accept that recording consultations is a product of the digital age. Patients may have many reasons for wanting to do so and it doesn't necessarily mean a lack of trust.

"They may not feel confident of remembering or fully understanding complex instructions or they may wish to share the information with their family to help them reach a decision on treatment options.

"You may wish to discuss their reasons so you can understand their rationale. If you feel uncomfortable at the prospect of being recorded, be honest and open, and tell them why. If a patient insists on recording a consultation, you should conduct the consultation as you normally would.

"It's our experience that most of these recordings support the actions of the doctor and confirm that they acted in an appropriate manner, both personally and clinically.

"It's possible that a dissatisfied patient may use a recording to pursue a complaint or claim, but doctors acting professionally should have nothing to fear. Keeping clear, comprehensive, and accurate written records of consultations and acting in accordance with the GMC's Good Medical Practice guidelines will help protect you if a patient tries to use the recording to support a complaint or claim."

\section{"Recordings can help patients take control"}

Adam R Jones, senior lecturer and severe haemophiliac, says, "What difference would it make to your practice if your patients were truly informed and were capable of self managing their conditions? What easier way could there be to achieve this than for patients to have access to recordings of conversations with their health professionals, for the purpose of clarification and understanding? After all, the patient is attempting to remember things they don't understand and trying to learn about their condition and how to treat it. They're trying their best to engage. This is often new for them-they want to learn so that they can feel in control of their illness and their life. Even if it's not new, they want to achieve self efficacy, even if they've never heard that word before.

"The request to record a consultation is not a hostile one, loaded with the intention of setting up barriers or conflict. Most patients are not familiar with medical terminology. They are in distress, and they want to listen and to understand, even though they're 
struggling. Ultimately, they want to be heard and understood. They want a two way conversation where their priorities count. So, let them record with no strings attached. I imagine doctors may worry about the possibility of litigation or being held to their word when they're exploring diagnoses or treatments outside of their specialism or comfort zone. But is this reality? I doubt it. In my experience, patients are much more forgiving than you may first think." 